\title{
Transfer of Gentamicin Resistance between Strains of Staphylococcus aureus on Skin
}

\author{
By JAY NAIDOO AND W. C. NOBLE \\ Department of Bacteriology, Institute of Dermatology, \\ St John's Hospital, Homerton Grove, London E9 6BX
}

(Received 13 February 1978; revised 30 March 1978)

\section{INTRODUCTION}

Transfer of antibiotic resistance between strains of Staphylococcus aureus has rarely been studied in vivo. In animals, Jarolmen et al. (1965) reported that a culture acquired tetracycline resistance from a lysate and Novick \& Morse (1967) reported transfer of a penicillinase plasmid between two cultures. Lacey (1971) demonstrated transfer of neomycin resistance between strains of $S$. aureus on healthy human skin under conditions closely resembling those occurring in hospital. Lacey \& Richmond (1974) reported the transfer of plasmids specifying penicillinase production and resistance to erythromycin, or resistance to neomycin and tetracycline, on skin of volunteers. We have studied the apparent acquisition of resistance to several antibiotics in patients with skin disease (unpublished observations). This paper reports studies on a pair of strains isolated from one such patient in which transfer of gentamicin resistance occurred with greater frequency after inoculation on to human and mouse skin than in laboratory culture media.

\section{METHODS}

Staphylococci. The strains (Table 1) were fresh isolates from a single patient. Each culture was purified thrice on nutrient agar. Donor and recipient fluid cultures were then prepared from single colonies.

Media. Oxoid blood agar base containing the appropriate antibiotic(s) at $10 \mu \mathrm{g} \mathrm{ml}^{-1}$ was used for the initial screening of antibiotic resistance. Disc sensitivity tests were carried out on Mueller-Hinton agar using Oxoid sensitivity discs by a modified Kirby-Bauer technique. Oxoid nutrient broth sometimes contained $0.01 \mathrm{M}-\mathrm{CaCl}_{2}$. Minimum inhibitory concentrations (m.i.c.) were determined by a plate method using doubling dilutions (gentamicin and streptomycin) or by the tube method using doubling dilutions (neomycin and kanamycin). Phage typing was carried out using the International set of typing phages with the addition of a new typing phage (no. 90) kindly supplied by the Staphylococcus Reference Laboratory, Central Public Health Laboratory.

Phage induction. Lysates were prepared by growing broth cultures to early-exponential phase at $37^{\circ} \mathrm{C}$ and adding mitomycin-C (Sigma) to a final concentration of 0.1 to $5 \mu \mathrm{g} \mathrm{ml}^{-1}$, depending on the strain. The cultures were incubated for a further $15 \mathrm{~min}$, centrifuged and the cells were resuspended in fresh warmed nutrient broth. After the culture cleared, the lysate was centrifuged and the supernatant was stored with chlcroform at $4{ }^{\circ} \mathrm{C}$.

Plasmid transfer. Gentamicin resistance was transferred from strain 4 (NT; non-typable) to strain 1 (77/84/90; gentamicin-sensitive but neomycin-resistant). Strain 4 (NT) was subcultured on agar containing $10 \mu \mathrm{g}$ gentamicin $\mathrm{ml}^{-1}$ because of the instability of the resistance (see below); strain 1 (77/84/90) was grown on agar without antibiotic. Both strains were suspended separately in broth to a density of about $10^{9}$ viable units $\mathrm{ml}^{-1}$. They were then applied either singly or together $(1: 1)$ to skin or incubated in broth. Broth cultures of the mixed strains were also incubated at dilutions giving counts of approximately $10^{8}, 10^{7}$ or $10^{8} \mathrm{ml}^{-1}$. Viable counts revealed that little or no multiplication occurred at $10^{8}$ or $10^{9} \mathrm{ml}^{-1}$, but that multiplication up to 20 -fold occurred at the lower dilutions. The presence of gentamicin-resistant recipients was detected by applying a total of $2.5 \mathrm{ml}$ of each broth culture to the surface of agar containing $10 \mu \mathrm{g}$ neomycin and $10 \mu \mathrm{g}$ gentamicin $\mathrm{ml}^{-1}$.

The ventral surface $(7 \times 5 \mathrm{~cm})$ of unprepared human forearm skin was occluded with sterile plastic film sealed to the skin with Steridrape (3M Company). An area $(5 \times 3 \mathrm{~cm})$ of the skin of the back of obese/ hairless mice, bred at the Institute of Dermatology (J. Atkinson, unpublished observations), was occluded in the same way. Cultures were applied using a cotton swab and the undiluted cultures were sampled subsequently using contact plates or soluble alginate swabs dissolved in buffer and inoculated on to agar 
containing gentamicin and neomycin. Dilutions of the recovered bacteria were also inoculated on to agar containing a single antibiotic, for viable count determinations.

\section{RESULTS AND DISCUSSION}

Characteristics of the strains recovered from the patient and from skin are shown in Table 1. The gentamicin-sensitive 77/84/90 strain (1) was isolated from the patient on the day of admission to hospital and the NT strain (3) was acquired 5 weeks later during her hospital stay. Gentamicin-resistant derivatives of both strains ( 2 and 4 ) were first isolated at about the same time ( 7 weeks after admission) during topical and systemic treatment of the patient with this drug. In each strain gentamicin resistance was labile. Despite three subcultures on agar containing $20 \mu \mathrm{g}$ gentamicin $\mathrm{ml}^{-1}$, a single subculture on to antibiotic-free agar yielded variants sensitive to gentamicin. Such labile gentamicin resistance has been reported previously (Soussy et al., 1975).

Gentamicin resistance was acquired by strain 1 after inoculation together with strain 4 on to two volunteers on each of three separate occasions. The results of a single experiment on the arms of seven laboratory workers and on four mice are shown in Table 2. No gain of resistance occurred after the inoculation of the strains singly on two occasions on two volunteers and two mice.

The direction of transfer was deduced as being from strain 4 to strain 1 since the gentamicin-resistant strain had the same properties as strain 1 (Table 1) apart from the acquisition of gentamicin resistance (m.i.c. $64 \mu \mathrm{g} \mathrm{ml}^{-1}$ ). However, no transfer occurred in the broth culture at any of the initial densities used. The gentamicin-resistant recipients (strain 5) had apparently become lysogenic (Table 1). The newly acquired gentamicin resistance was as labile as that in strain 4 and the gentamicin-resistant variant of strain 1 isolated from the patient (strain 2). Thus, if gentamicin resistance is plasmid-mediated in these strains, its instability is probably a feature of the plasmid rather than the chromosome. Failure of transfer of gentamicin resistance from a phage lysate of strain 4 to strain 1 either inoculated on skin or in broth containing $0.01 \mathrm{M}-\mathrm{CaCl}_{2}$ could be due to the low titres of phage obtained ( $10^{4}$ plaque-forming units $\mathrm{ml}^{-1}$ titrated on the $77 / 84 / 90$ strain). Alternatively, the transducing phages may be short-lived and/or cell-bound (Lacey \& Richmond, 1974). Although Witte (1977) proposes that cell-to-cell contact may be a means of transfer in $S$. aureus, phage particles seem a more likely mechanism.

Although the mechanism of transfer has not been determined in these experiments, the results emphasize the epidemiological potential of the skin in that antibiotics can interact

\section{Table 1. Characteristics of S. aureus strains}

$\begin{array}{clllc}\text { Strain } & \text { Source } & \begin{array}{c}\text { Phage typing } \\ \text { pattern } \\ \text { (RTD)* }\end{array} & \begin{array}{l}\text { Resistance } \\ \text { pattern } \dagger\end{array} & \begin{array}{c}\text { Lysogenic } \\ \text { pattern } \ddagger\end{array} \\ 1 & \text { Patient } & 77 / 84 / 90 & \text { PTNE } & - \\ 2 & \text { Patient } & 77 / 84 / 90 & \text { PTNEG } & - \\ 3 & \text { Patient } & \text { NT } & \text { PTESM } & + \\ 4 & \text { Patient } & \text { NT } & \text { PTESMG } & + \\ 5 & \text { Laboratory volunteers and mice } & 77 / 84 / 90 & \text { PTNEG } & +\end{array}$

$*$ NT, Not typable using standard set of phages at routine test dilution (RTD) and $100 \times$ RTD following incubation at 37 or $42^{\circ} \mathrm{C}$.

$\dagger \mathrm{P}$, Resistant to penicillin (penicillinase production); $\mathrm{T}$, resistant to tetracycline; $\mathrm{N}$, resistant to neomycin (m.i.c. $64 \mu \mathrm{g} \mathrm{ml}^{-1}$ ); E, dissociated resistance to erythromycin; $\mathrm{S}$, very high level of resistance to streptomycin (m.i.c. $>1000 \mu \mathrm{g} \mathrm{ml}^{-1}$ ); $\mathrm{M}$, resistant to methicillin $\left(\right.$ at $30^{\circ} \mathrm{C}$ ); $\mathrm{G}$, resistant to gentamicin (m.i.c. $>64 \mu \mathrm{g} \mathrm{ml}^{-1}$ ). Gentamicin-resistant variants were also resistant to kanamycin and tobramycin. Strain 3 was sensitive to neomycin (m.i.c. $<2 \mu \mathrm{g} \mathrm{ml}^{-1}$ ) and kanamycin (m.i.c. $<1 \mu \mathrm{g} \mathrm{ml}^{-1}$ ). Strain 4 (resistant to gentamicin) was also sensitive to neomycin (m.i.c. $<2 \mu \mathrm{g} \mathrm{ml}^{-1}$ ) but resistant to kanamycin (m.i.c. $>128 \mu \mathrm{g} \mathrm{ml}^{-1}$ ).

$\ddagger+$, All strains were induced to yield phage lytic for phage propagating strains (PS) Ps6, Ps47, Ps75, Ps77, PS83A and the wild 77/84/90 strains; -, non-lysogenic. 
Table 2. Recovery of gentamicin-and neomycin-resistant $S$. aureus from skin of seven humans and four mice following incubation of mixed culture of strains 1 and 4

\begin{tabular}{|c|c|c|c|}
\hline $\begin{array}{l}\text { Volunteer/ } \\
\text { mouse }\end{array}$ & $\begin{array}{l}\text { Period of } \\
\text { occlusion } \\
\text { (h) }\end{array}$ & $\begin{array}{c}\text { Total } S \text {. aureus } \\
\text { population recovered } \\
\text { from skin }\end{array}$ & $\begin{array}{l}\text { No. of } \mathbf{G}+\mathbf{N} \\
\text { variants } \\
\text { recovered* }\end{array}$ \\
\hline \multicolumn{4}{|l|}{ Human: male } \\
\hline 1 & 0 & $2 \times 10^{5}$ (R. arm) & 0 \\
\hline 1 & 4 & $3 \times 10^{6}$ (R. arm) & 12 \\
\hline 1 & 6 & $3 \times 10^{6}$ (L. arm) & 12 \\
\hline 2 & 6 & $2 \times 10^{6}$ & 0 \\
\hline 3 & 6 & $2 \times 10^{6}$ & 8 \\
\hline \multicolumn{4}{|c|}{ Human: female } \\
\hline 1 & 4 & $2 \times 10^{6}$ (R. arm) & 6 \\
\hline 1 & 6 & $5 \times 10^{6}$ (L. arm) & 3 \\
\hline 2 & 6 & $3 \times 10^{6}$ & 4 \\
\hline 3 & 6 & $4 \times 10^{6}$ & 0 \\
\hline 4 & 6 & $7 \times 10^{8}$ & 8 \\
\hline \multicolumn{4}{|l|}{ Mouse } \\
\hline 1 & 4 & $8 \times 10^{5}$ & 0 \\
\hline 2 & 4 & $2 \times 10^{6}$ & 2 \\
\hline 3 & 6 & $2 \times 10^{6}$ & 6 \\
\hline 4 & 6 & $4 \times 10^{6}$ & 4 \\
\hline
\end{tabular}

$*$ Number of gentamicin- and neomycin-resistant variants growing on plates. As only $10 \%$ of the inocu-
lum recovered from skin was used on antibiotic plates, estimated recovery is 10 -fold greater than actual.

with the organism in its natural environment (Lacey, 1975; Noble \& Naidoo, 1978). We have shown here that transfer of gentamicin resistance can occur between strains of $S$. aureus inoculated on human and mouse skin in the absence of antibiotic therapy under conditions which closely resemble those in dermatology patients undergoing treatment. If transfer occurred commonly, topical or even systemic antibiotics would select large numbers of new resistant derivatives. It remains to be seen whether it is more than mere coincidence that Lacey's strains, which gave high frequency transfer to neomycin resistance, and our own, which gave high frequency transfer of gentamicin resistance, were both from patients in dermatology wards and were resistant to similar antibiotics, both of which are used topically.

Jay Naidoo is indebted to the Wellcome Trust for support.

\section{REFERENCES}

JARolmen, H., Bondi, A. \& Crowell, R. L. (1965). Transduction of Staphylococcus aureus to tetracycline resistance in vivo. Journal of Bacteriology 89, 1286-1290.

LACEY, R. W. (1971). High frequency transfer of neomycin resistance between naturally occurring strains of Staphylococcus aureus. Journal of Medical Microbiology 4, 73-83.

LACEY, R. W. (1975). Antibiotic resistance plasmids of Staphylococcus aureus and their clinical importance. Bacteriological Reviews 39, 1-32.

LACEY, R. W. \& Richmond, M. H. (1974). The genetic basis of antibiotic resistance in $S$. aureus: the importance of gene transfer in the evolution of this organism in the hospital environment. Annals of the New York Academy of Sciences 236, 395-412.
Noble, W. C. \& NaIdoo, J. (1978). Evolution of antibiotic resistance in Staphylococcus aureus: the role of the skin. British Journal of Dermatology 98, 481-489.

NoviCK, R. P. \& Morse, S. I. (1967). In vivo transmission of drug resistance factors between strains of Staphylococcus aureus. Journal of Experimental Medicine 125, 45-59.

Soussy, C. J., Bouanchaud, D. H., Fouace, J., Dublanchet, A. \& Duval, J. (1975). A gentamicin resistance plasmid in Staphylococcus aureus. Annales de Microbiologie (Institut Pasteur) 126B, 91-94.

WITTE, W. (1977). Transfer of drug-resistanceplasmids in mixed cultures of staphylococci. Zentralblatt für Bakteriologie und Hygiene (Abteilung I, Originale A) 237, 147-159. 